\title{
Green-Wave Traffic Theory Optimization and Analysis
}

\author{
Xiaoping Wu${ }^{1}$, Shuai Deng ${ }^{1}$, Xiaohong $\mathrm{Du}^{2}$, Jing Ma1 \\ ${ }^{1}$ School of Civil Engineering \& Mechanics, Huazhong University of Science and Technology, Wuhan, China \\ ${ }^{2}$ China Huaxi Engineering Design \& Construction CO., LTD Wuhan Branch, Wuhan, China \\ Email: 652485344@qq.com
}

Received May 2014

\begin{abstract}
This paper analyzed the applicable conditions of the Green-Wave traffic theory, used two-phase signal control concept to optimize the Green-Wave traffic theory, put forward specific program for cross intersections and T-intersections. The analysis concluded that the optimized Green-Wave traffic theory is favorable to improve road safety and reduce vehicle fuel consumption and reduce vehicle emissions and other aspects.
\end{abstract}

\section{Keywords}

\section{Green-Wave Traffic, Two-Phase Signal Control, Road Safety, Energy Conservation and Emission} Reduction, Intersection

\section{Introduction}

With traffic mechanization, more and more problems are exposed in traditional road traffic design methods. According to the statistics, in 2013, a total of 667,507 road traffic accidents occurred in China, 494,174 people injured, the death toll reached 104,342. In terms of energy consumption, in the United States, the transportation system consumed $60 \%$ of the total fuel, of which $73 \%$ were consumed by motor transport [1]; currently, transportation system energy consumption accounted for about $20 \%$ of China's total energy consumption [2]. In addition, vehicle exhaust emission of the road transport system is an important source of air pollution.

Green-Wave traffic is an overall traffic control concept, the whole road's intersections are coordinated and controlled by signal lights to ensure road safety and traffic efficiency. In this paper, two-phase traffic signal control is used to optimize the Green-Wave traffic theory, so as to provide better travel environment for pedestrians and vehicles.

For safety, energy conservation and emission reduction, this paper compared road traffic signal design before and after optimization, and the results showed that the Green-Wave traffic optimized by two-phase signal control concept is more conducive to road safety and energy-saving and emission reduction.

\section{Green-Wave Traffic Theory Optimization}

\subsection{Green-Wave Traffic}

"Green-Wave” refers to signal multi-point control technique, namely, implement uniform signal lights control 
within a traffic area or along a traffic route, all signal lights within the control range will be incorporated into the computer control system. Seeing from color of the signal lights at each intersection along the controlled arterials, the green lights are rolling forward like waves, so it is vividly called "Green-Wave" [3]. The vast majority of vehicles that Green-Wave serves will encounter the green light when arriving at the intersections.

\subsection{Green-Wave Traffic Applicable Conditions}

Reasonable implementation of the Green-Wave traffic will improve traffic efficiency, but in some cases, the result may be counterproductive-reducing traffic efficiency, causing traffic hazards. The suitability of GreenWave traffic generally needs to consider several governing factors [3]:

1) Vehicle arrival characteristics. Compared to the uniform traffic, the impulse-type traffic is more inclined to form vehicle queue at intersections, which is more suitable for the implementation of the Green-Wave traffic.

2) Traffic organization. The simpler form of traffic organization is better, for example, one-way traffic organization is more conducive to the implementation of Green-Wave traffic. Meanwhile, the fewer traffic flow directions or bigger difference in traffic flow distribution of each direction is more suitable for the implementation of the Green-Wave traffic.

3) Adjacent intersection spacing. Proper spacing between adjacent intersections is conducive to intersection signals coordination. In general, the larger distance between intersections, the worse line control effects. However, spacing between intersections should not be too small, because smaller intersection spacing is easier to form a uniform traffic.

4) Signal phase setting condition. Signal phase has a great impact on the implementation of Green-Wave traffic. The fewer signal phases, the better implementation of the Green-Wave traffic, and the greater bandwidth of Green-Wave band.

\subsection{Green-Wave Traffic Theory Optimization}

According to the above analysis, implementation of the Green-Wave traffic needs to consider vehicle arrival characteristics, traffic organization condition, intersection spacing, signal phase setting condition. As vehicle arrival characteristics belongs to traffic flow study field, it will not be discussed in this paper.

1) Use two-phase signal control concept to improve traffic organization. Adopt two-phase signal control, prohibit left turn at intersections (traffic volume is small), only straight traffic (main traffic direction) is remained, to reduce the traffic flow direction within the intersection, other traffics are achieved by bypassing and U-turn. Such simplified traffic organization mode is more suitable for the implementation of the Green-Wave traffic.

2) Use two-phase signal control concept to adjust the intersection spacing. In order to meet requirements of intersection spacing and its uniformity for Green-Wave traffic, the two-phase method takes signals spacing as the main parameter, intersection spacing is adjusted from the point of signal setting, i.e., on the basis of current road intersections, set signal phase appropriately, combined with left turn forbidding, so that distance between each control signal is maintained at an appropriate level to facilitate the implementation of the Green-Wave traffic.

3) Use two -phase signal control to simplify the signal phase. Only two phases are retained along the whole road, to simplify phase setup. There is two-phrase decreasing of cross intersection, and one phase decreasing of T-intersection, which facilitates the implementation of the Green-Wave traffic.

According to the previous analysis, specific organization solutions of T-intersection and cross t intersection are designed, as shown in Figure 1 and Figure 2.

\subsection{Advantages and Disadvantages of the Program}

\subsubsection{The Above Program Has the Following Advantages}

1) People-oriented

Traditional designs are often vehicle-oriented, and vehicle running is taken as a controlling factor in the design of intersection, while ignoring the safety factor of pedestrian crossing, so the phenomenon of pedestrian running across the street is often seen, especially for the elderly, children and other vulnerable groups, there is a serious safety hazard while walking across the street. 


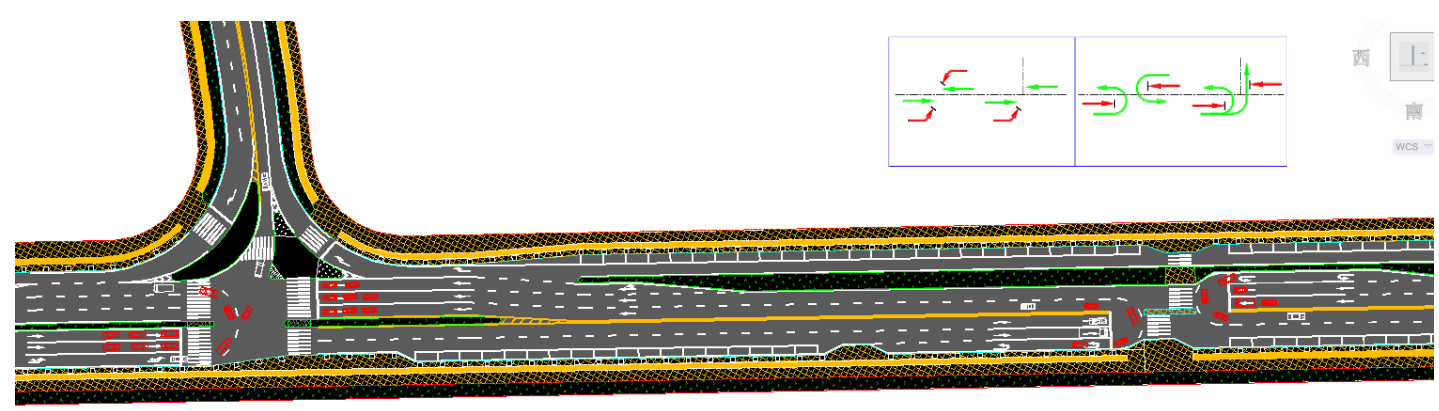

Figure 1. Optimized Green-Wave traffic phase diagram (T-type intersection).

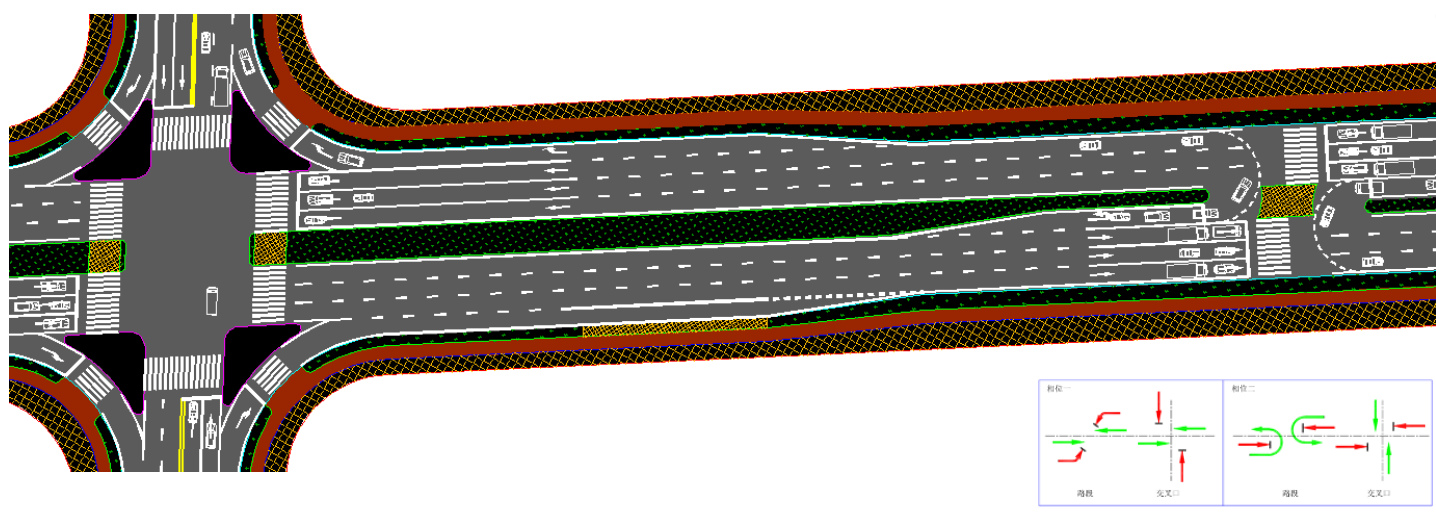

Figure 2. Optimized Green-Wave traffic phase diagram (cross intersection).

Optimized Green-Wave traffic theory is people-oriented, taking pedestrian and vehicles into equal consideration. As shown in Figure 3, pedestrian crossing and vehicles U-turn are achieved in the same phase, and enough distance is set between crosswalk and track of vehicle U-turn. By the dislocation setting of crosswalk and setting two-step crossing safety island, and expanding stand stone space (road side) to shorten the crossing time of pedestrians, the optimized system is safer.

2) Safer for car

The intersection is a traffic hazard place and accident-prone location. View from macro perspective, around the world, about $30 \%$ to $50 \%$ of road traffic accidents occur at intersections [4]. From the microscopic point of view, the proportion of accidents happened in each flow direction at intersections is different. As shown in Figure 4, taking T-intersection for example, according to statistical data collected by the US Federal Highway Administration in 2006, within the T-intersections, the number of accidents happened at six traffic flows directions is very different, of which, the accident rate of two straight traffic flows on the main road is small, and for the remaining four flows, left turn at the main road is of the largest traffic accident rate, accounting for $47 \%$, followed by left turn on the secondary road, accounting for $27 \%$. Right turn on the main road and secondary road accounted for $16 \%$ and $10 \%$ respectively. Therefore, probability proportion of car accident of left turn, right turn and straight vehicle in the T-intersections can be concluded: left turn > right turn > straight.

In optimized Green-Wave traffic theory, according to probability difference of accidents occurred in different steering behaviors, two-phase signal control theory is used for intersection phase further optimization. Turn left at the secondary road is not achieved at the current intersection, but transferred to the road or adjacent intersection, achieved through a dedicated U-turn signal phase, at the same time providing sufficient turning radius for vehicles U-turn, in theory, can eliminate accidents of U-turn. Thus, only remain the straight flow and U-turn phase along the whole road. According to the steering accident probability data, vehicles' left turn behavior is very unfavorable for road safety. In the optimized Green-Wave traffic theory, to meet the needs of vehicle left turn by means of "go straight first and then make U-turn", has largely improved road safety.

\subsubsection{Program "Defects"}

This program forces left-turning vehicles to make detour, which seems to increase fuel consumption and emis- 


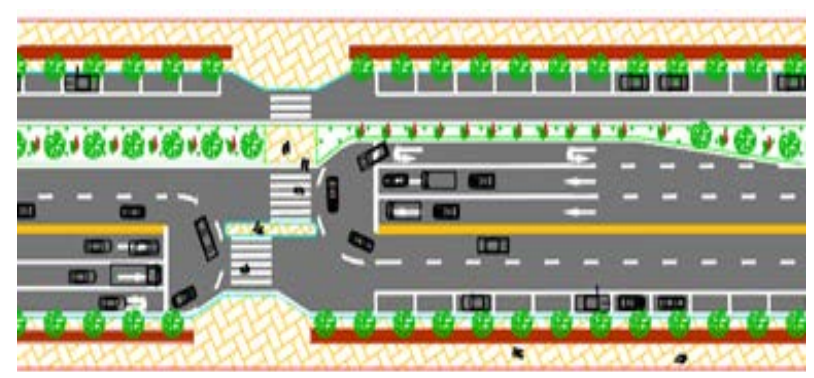

Figure 3. Pedestrian crossing diagram.

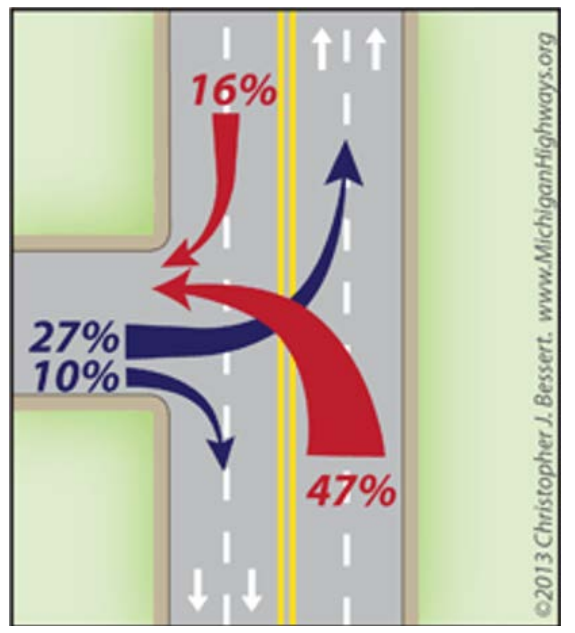

Figure 4. T-type intersection accidents distribution.

sions of left-turning vehicles, thus increasing the operating cost of the entire transport system.

\section{Traffic Transportation Efficiency Analysis}

For the concept of traffic transportation efficiency, Cai Jun [5] defines it as the effective transport tasks completed in per unit area of road, within a certain range of time-consuming. In a broad sense, it can be interpreted as effective transport tasks completed at the cost of the actual unit cost, time, energy consumption and economic depletion and damage to the environment, etc. is collectively defined as the cost.

Since the implementation of the Green-Wave traffic will force part of vehicles to detour, it will increase fuel consumption and exhaust emissions, reduce efficiency superficially, but in fact, the use of the optimized GreenWave traffic theory is conducive to transportation energy saving and emission reduction.

\subsection{Vehicle Fuel Consumption Analysis}

Vehicle fuel consumption is one of the most typical transportation costs. Reducing vehicle fuel consumption, on the one hand, can save individuals' travel costs; from macro perspective, it is also beneficial for worldwide energy conservation. Factors affecting motor vehicles fuel consumption, include age, mileage, emissions control technology, driving conditions, outside temperature and altitude, etc. [6]. Fuel consumption analysis in this paper mainly targets for the following two questions: 1) relationship between vehicle fuel consumption and its driving state; 2) relationship between vehicle fuel consumption and its speed. Based on these questions, collect data on the Internet and organize them into Table 1, the unit is constant fuel consumption per hundred kilometers.

Table 2 is obtained after treatment of Table 1, the unit is fuel consumption per hour.

For Questions 1: after analysis of data in Table 2, it shows that the fuel consumption in the idling state may be much higher than that of the constant speed state, for example, Civic series cars, in fact, idling fuel consump- 
Table 1. Several cars' idling fuel consumption and constant speed fuel consumption (collect from the Internet).

\begin{tabular}{|c|c|c|c|c|c|c|c|c|c|c|}
\hline \multirow{2}{*}{ Car } & \multirow{2}{*}{$\begin{array}{l}\text { idling fuel } \\
\text { consumption }(\mathrm{L} / \mathrm{h})\end{array}$} & \multicolumn{9}{|c|}{ constant speed fuel consumption $(\mathrm{L} / 100 \mathrm{~km})$ at corresponding speed $(\mathrm{km} / \mathrm{h})$} \\
\hline & & 40 & 50 & 60 & 70 & 80 & 90 & 100 & 110 & 120 \\
\hline Civic & 4.35 & 5.31 & 4.73 & 4.41 & 4.53 & 4.84 & 5.21 & 5.87 & 6.42 & 7.15 \\
\hline Sagitar & 2.72 & 6.07 & 5.47 & 5.88 & 5.71 & 6.37 & 6.93 & 7.78 & 8.42 & 9.12 \\
\hline Camry & 3.0 & 5.65 & 5.8 & 5.89 & 6.03 & 5.57 & 6.15 & 6.14 & 6.97 & 7.69 \\
\hline
\end{tabular}

Table 2. Several cars’ idling fuel consumption and constant speed fuel consumption (L/h).

\begin{tabular}{|c|c|c|c|c|c|c|c|c|c|c|}
\hline \multirow{2}{*}{ Car } & \multirow{2}{*}{$\begin{array}{c}\text { idling fuel } \\
\text { consumption (L/h) }\end{array}$} & \multicolumn{9}{|c|}{ constant speed fuel consumption $(\mathrm{L} / 100 \mathrm{~km})$ at corresponding speed $(\mathrm{km} / \mathrm{h})$} \\
\hline & & 40 & 50 & 60 & 70 & 80 & 90 & 100 & 110 & 120 \\
\hline Civic & 4.35 & 2.12 & 2.37 & 2.65 & 3.17 & 3.87 & 4.69 & 5.87 & 7.06 & 8.58 \\
\hline Sagitar & 2.72 & 2.43 & 2.74 & 3.53 & 4.00 & 5.10 & 6.24 & 7.78 & 9.26 & 10.94 \\
\hline Camry & 3 & 2.26 & 2.32 & 2.36 & 2.41 & 2.23 & 2.46 & 2.46 & 2.79 & 3.08 \\
\hline
\end{tabular}

tion is measured at $4.35 \mathrm{~L} / \mathrm{h}$, and when it when driving at $40 \mathrm{~km} / \mathrm{h}$ constant speed, the fuel consumption is only $2.12 \mathrm{~L} / \mathrm{h}$, less than half of the idling fuel consumption.

For Questions 2: JIA Hong-fei et al. [7] study found, a fuel-speed model is built for the highway fuel consumption, this article will apply it to urban road. In this model, the car's fuel consumption is a quadratic function of speed, namely:

$$
F=a v^{2}+b v+c
$$

where: $F$ is the basic automotive fuel $\mathrm{L} / 100 \mathrm{~km} ; v$ is vehicle speed, $\mathrm{km} / \mathrm{h} ; a, b, c$ are regression coefficients.

According to the above expression, when running at a certain constant speed, the basic fuel consumption is at minimum, then the speed is the vehicle's economic running speed, in fact, from the energy point of view, it may be referred to as energy-saving speed. According to analyzes obtained from Table 1, different vehicle models have different economic speed, keep the speed at the vicinity of the economic speed, is advantageous to reduce fuel consumption.

\subsection{Vehicle Emission Analysis}

Vehicle exhaust emissions analysis usually takes CO, HC, NOx as the main indicator. Different vehicles have different exhaust emissions, and there are also emission differences in the same vehicle under different driving conditions. Vehicle running situations are actually a combination of different proportions of idling, acceleration, deceleration and constant speed state. Ma Dong et al. [8] conducted experiment and got relevant conclusion: When the car traveling at a low speed, emission factors are generally high, when the average speed increases, factors will decline. When idling and deceleration driving conditions are of high proportions, emission factors are relatively high; when constant speed driving condition is of higher proportion the emission factor is relatively low.

\subsection{Significance of Optimized Green-Wave Traffic Theory for Energy Conservation and Emission Reduction}

Encountering the red traffic light, vehicles will slow down and stop before the stop line, and then remain idle waiting for the green signal, finally accelerate to leave the intersection after the traffic light turns green, the whole process involves vehicle states of acceleration, idling, deceleration. According to the above analysis, in such driving conditions, constant speed state is of small proportion, which is not conducive to energy conservation and emission reduction. In order to avoid the above situation, UPS (United Parcel Service) prohibitsits transport vehicle turning left at intersections, and detouring to achieve the purpose of left turn, then the company saved a total of ten million gallons of gasoline from 2004 to 2009, reduced one hundred thousand tons of carbon emissions, equivalent to emissions of 5300 cars a year.

The practical results of UPS coincide with optimized Green-Wave traffic theory. At traditional three-phase 
T-intersections with signals, the probability of vehicle stop is $2 / 3$, according to the theory of probability and statistics, about $2 / 3$ of all vehicles passing through this intersection will stop driving, which will reduce the proportion of constant speed state. While in two-phase signal control theory, intersections along the whole section are provided with only two phases, reduce the probability of vehicle stop to $1 / 2$, while green light ratio will be improved, reducing the probability of stopping, thereby proportion of vehicles at constant speed driving conditions under two -phase signal controlling increases, so as to achieve the purpose of energy saving and emission reduction. Therefore, the optimized Green-Wave traffic theory is effective.

\section{Conclusion}

After analysis and demonstration, compared to the traditional signal phase design, the Green-Wave traffic theory optimized by two-phase signal control is more rational design, and more favorable for reducing vehicle operating fuel consumption, reducing emissions, and improving the safety of all road intersections.

\section{References}

[1] Zhang, W.H., Wang, W. and Hu, G. (2003) Low Transport Energy Consumption-Based Urban Development Strategy. Highway and Transportation Research, 2, 80-84.

[2] Wang, W., et al. (2003) Urban Transport System Energy Consumption and Environmental Impact Analysis Method. Science Press, Beijing, 70-74.

[3] Xu, B.Y. (2012) Applicability Study of Urban Main Road Green Wave Band Control. Nanjing Forestry University.

[4] Pan, F.Q., Zhang, L.X., Lu, J. and Wang, F.Y. (2013) Computation Module of Vehicle Conflict Points at No SignalControlled Intersections. Journal of Shanghai Jiao Tong University, 2.

[5] Cai, J. (2013) Urban Road Network System Planning. Chinese Building Industry Press.

[6] Wang, N.N. (2012) Urban Road Driving Conditions Building and Fuel Consumption Research. Hefei University of Technology.

[7] Jia, H.F., Juan, Z.-C., Zhang, X.X., and Nian, A.N. (2004) Highway Fuel Consumption Evaluation Indicators Identification and Comparison.

[8] Ma, D., Zhao, Y. and Liang, B. (2010) Study of Lightweight Vehicle Emission Characteristics under Different Driving Conditions. Beijing Automotive, 4. 